\title{
Fallback to SRB for Network Optimization in Small Cells
}

\author{
Rajkiran S. N. \\ Lead Engineer, \\ Radisys India Private Limited, \\ Bangalore, Karnataka.
}

\begin{abstract}
In this modern era computers and mobile phones are the major mode of communication. Due to the rapid development in mobile phone technology, which leads to smart phones with tremendous applications installed on it. This made wide impacts in our business as well as social lives. The advancement of new generation smartphones are capable of handling both voice and data service simultaneously in a cellular network through the same antenna. This requires better radio connectivity compare to voice alone service. This paper converses the concepts of Fallback to Signaling Radio Bearers for network optimization in Macro cell deployments and Small-Cells which includes Femto, Pico and Micro cells. It discusses the advantages of using Fallback to Signaling Radio Bearers (Fb-SRB) over the NBAP (Node B Application Part) signaling mechanism executed to camp-on to the network and macro-cell based as well. It also talks about the factors improving the performance of Fallback to Signaling Radio Bearers (Fb-SRB).
\end{abstract}

\section{General Terms}

Signaling lost generally refers to the phenomenon of call/packet dropping in both voice and data networks. Call/packet dropping refers to the event described as the termination of calls in progress before either involved party intentionally ends the call. Wireless networks involve radio and wire-line links as well as switching hardware and software, and data base operations. However, drop call rate is mainly determined by the radio resources in the network.

Wireless networks involve radio and wire-line links as well as switching hardware and software, and data base operations. However, drop call rate is mainly determined by the radio resources in the network. These resources translate mainly into the plethora of radio channels all of which share a common bandwidth through a process known as frequency reuse

\section{Keywords}

SRB, NBAP, HNB, MRAB, Call Drop, Small Cells.

\section{INTRODUCTION}

The wide usage of cell phones has increasing impacts in our social and business lives. Numerous data applications have emerged with the rapid advancement of new-generation smartphones. Cisco suggests that the average monthly data usage of the smartphone nearly tripled in 2011, and will increase 17 -fold by 2020 [1]. Thus, supporting simultaneous

Voice and data services in a cellular network becomes not only desirable, but also indispensable with the advent and increasing popularity of the feasible network topology architecture for better capacity, coverage and better last mile experience, the network operators are keenly derived towards the small cell architecture to satisfy the growing demand of the end user's customer premises equipment. In this regards small cells are fully featured, short range mobile phone base stations used to complement mobile phone service from larger microcell towers. These are connected using standard domestic internet broadband through to larger equipment used inside commercial offices or outdoor public spaces. They offer excellent mobile phone coverage and data speeds at home, in the office and public areas for both voice and data. Small cells have been developed for both $3 \mathrm{G}$ and the newer 4G/LTE radio technologies. However the network operators are challenged with a number of practical deployments issues on either side of Base Station Transceivers as well as Customer Premises Equipment's hardware and its composition for better reliability and battery power consumption as in our case the smart phones.

Equipped with only one radio antenna in most of the $3 \mathrm{G}$ smartphones to transmit voice and data through cellular network. Current 3G networks are using CDMA2000/ EVDO [1] \& [2] technology, where voice and data are handled separately, i.e., when a voice call comes, then the data sessions will be blocked. 3G networks with UMTS (Universal Mobile Telecommunication System) [3] provides the appealing feature of supporting simultaneous voice and data by combining their packets together and sending over the single radio connection. For the new deployment of the $4 \mathrm{G}$ network such as LTE (Long Term Evolution) Advanced [4], provisioning voice over IP (VOIP) is not as mature as using the $3 \mathrm{G}$ techniques. Therefore, once a voice phone call is initiated, many carriers will switch the data sessions on LTE network to the mixed calls (voice + data) in $3 \mathrm{G}$ network by utilizing Circuit Switched Fall Back (CSFB) [5].

To minimize dropped calls, one promising direction is to voluntarily block data sessions when the voice call could be dropped due to weak signals. Thus, the simplest call management strategy is to block data whenever a voice call is made, however, defeating the purpose of serving voice and data simultaneously. Another straightforward strategy [7] involves blocking data only when the receiving signal strength of a cell phone is less than a pre-specified threshold.

However, there is a no systematic approach of predicting potential call drops and intelligently performing call management by taking into account of users' satisfaction when supporting voice and data simultaneously. The major reasons of the dropped call in transmitting both voice and data based on a large carrier's UMTS network recording data, the aim at devising a smart call management strategy grounded on machine learning approaches that can predict drop calls by identifying most relevant features and improve the reliability of serving voice and data simultaneously via intelligent call management based on user satisfaction. Supporting reliable simultaneous voice and data services in UMTS networks will remain important in the future. However, it is known that the mixed calls require better radio connection than voice-only calls in UMTS networks [6], which demands the coordination between voice and data transmissions in case of weak signals. 
It is critical to reduce dropped calls and improve the service reliability when supporting voice and data simultaneously. The paper discusses on how Barb is advantageous over the implicit flow of signalling exchange mechanism that happens between the UE and the Node B.

\section{STUDY OF THE PROPOSED MODEL}

\subsection{NBAP Signaling Procedure}

In this section predominately it covers basic NBAP signaling procedures exchanged between the L1 (Node B) and Collapsed RNC (Small cell architecture). NBAP stands for 'Node B Application Part'. Based on the functionality, again subdivided into Common and Dedicated NBAPs. Over all Node B functionalities are handled by Common NBAP and radio links are controlled by dedicated NBAP especially for specific user equipment.

A Signaling Radio Bearer (SRB) is a radio bearer that carries DCCH signaling data. An SRB is used during connection establishment to establish the Radio Access Bearers (RABs) and then also to deliver signaling while on the connection (for example, to perform a handover, reconfiguration or release).

The following signaling services are studied: new call request, paging, location update, handover, SMS, and end call requests. Here to illustrate the procedures of these signaling services through their message flows. The signaling message length is also given for the purpose of the calculation in the code acquisition time. First, we consider the signaling services that affect the quality of the active user-data transmission on the up-link direction (i.e., location update, call setup, handover, and SMS). The user equipment (UE) must perform a general packet radio service (GPRS) attach, the security related procedures, and the packet data protocol (PDP) context before sending the data if any. The GPRS attach allows the system to handle the mobility management and to obtain the detailed location information. The PDP context characterizes sessions and assigns the PDP address for each PDP session. These procedures are illustrated in Figure 1. 2. RRC connection request complete (26 bytes) 1 . UE sends RRC connection request message over DCH/CCH (10 bytes) 4. Authentication and ciphering request (53 bytes) UE RNC SGSN HLR 6. Inform HLR to update UE's locations with SGSN number and SGSN address 7. Insert subscriber data 8. Validate UE' RA, MM context, insert subscriber data ACK to HLR VLR 5. Authentication and ciphering response (27 bytes) 11. Activate PDP context request ( 84 bytes) 13. Radio bearer setup (105 bytes) 10. Service accept (e.g., call setup 11 bytes, location update - 21 bytes, SMS) Security procedures MM context (Location update for first attach) 68100 bytes 3. Service request (e.g., call setup - 27 bytes, location update - 29 bytes, SMS - 1 to 100 Kbytes 2. RRC connection setup (139 bytes) 14. Radio bearer setup complete ( 7 bytes) 15. Activate PDP context accept (31 bytes) 16. Deactivate PDP context request (18 bytes) 17 . Deactivate PDP context accept (11 bytes) 19. Radio bearer release request ( 91 bytes) 20. Radio bearer release complete ( 7 bytes) 22. RRC connection release complete ( 2 bytes) 21 . RRC connection release (3 bytes) 9. Cancel old MM context, send location update ACK to SGSN 12. Radio bearer assignment 18. Radio bearer release Send data traffic Fig. 1. The GPRS attach and a PDP context [, these signaling procedures consist of the following steps. In step 1 , the radio resource control (RRC) connection is established over the $\mathrm{CCH}$. Then, in step 2 , the radio network controller (RNC) sets up a point-to-point radio connection as well as the signaling connection to the network before sending acknowledgment back to the UE.
After that, the UE will start the attach process in steps 3-10 which includes the attach request, the identity request/response for the first time that the UE is attached to the network, the authentication request/response if the mobility management context does not exist for the UE anywhere else. Then, the PDP context will be setup to characterize the radio bearer (RAB) session and RAB request is setup in step $11-15$. The PDP addresses that will be used and stored at the UE and the GPRS supported nodes (GSNs) are activated. The PDP context contains mapping and routing information for packet transmission between the UE and the gateway GSN (GGSN). After the UE finished data transmission, the RAB release is initiated along with the PDP context deactivation and the RRC release in step $16-22$.

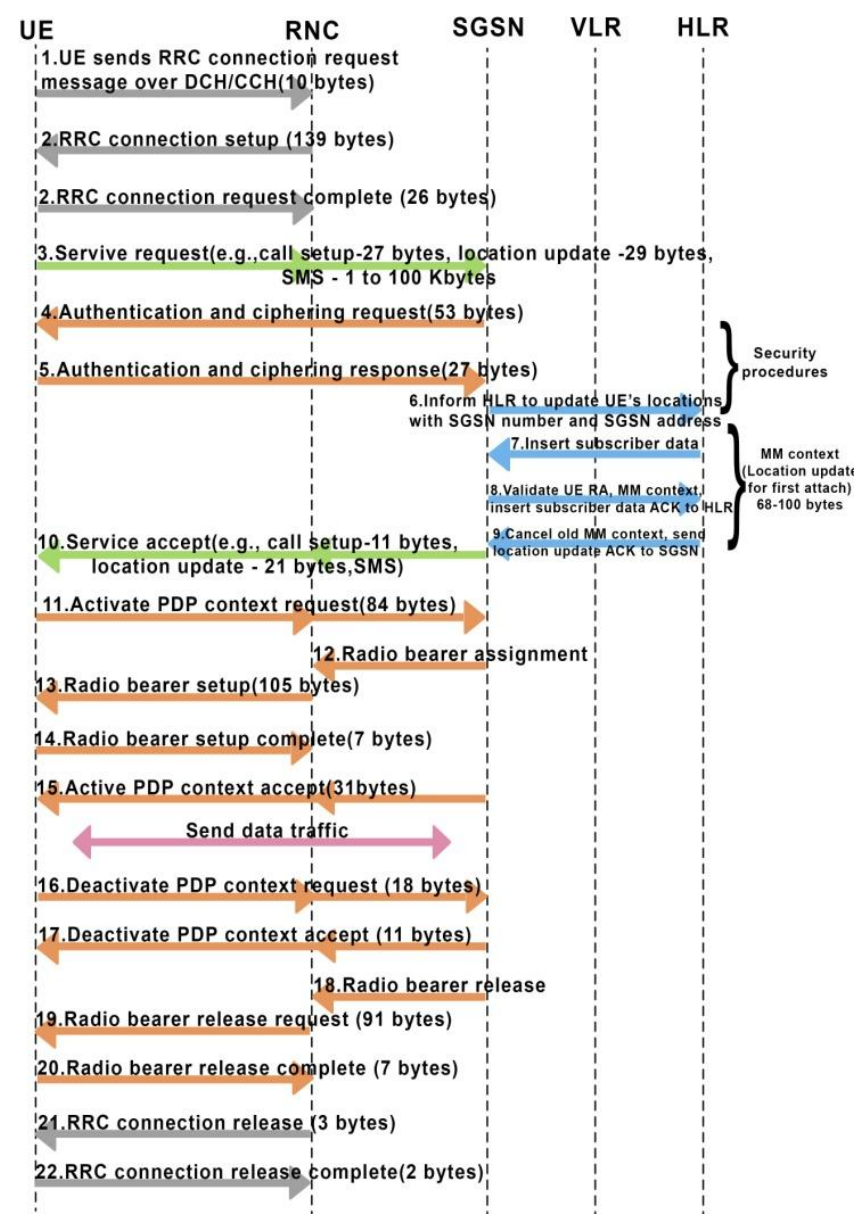

\section{Figure 1: The call flow of exchange of messages between User Equipment and the complete UMTS network elements}

Figure: 1 summarizes the acquisition time which can be derived from the total message length according to [9], and the channel data rate. LU considered here is the periodic LU where GPRS attach and security command are not performed. We use the maximum length of SMS message, 1Kbytes. Most of signalling services can be delivered over either the $\mathrm{CCH}$ or the $\mathrm{DCH}$, leading to the different code acquisition time. The $\mathrm{CCH}$ benefits from fast transmission since it does not require call setup or tear-down, and the ability to share code. Also, the interference is introduced only when the signalling services is transmitted, not in the idle period unlike in DCH. However, it lacks of fast power control which anticipates higher interference than $\mathrm{CCH}$. On the other hand, the $\mathrm{DCH}$ allows fast power control, but the interference is always generated 
even when channel is idle. According to the study in [9], the $\mathrm{CCH}$ is more suitable to lower burst size compared to the $\mathrm{DCH}$. More specifically, the $\mathrm{CCH}$ performs better than $\mathrm{DCH}$ for a signaling service session which transmits signaling messages of size approximately up to 250 bytes. Because the $\mathrm{CCH}$ access time is shorter than the setup time of DCH. In the up-link, the maximum data rate for the $\mathrm{CCH}$ and $\mathrm{DCH}$ are 60 kbps and $48 \mathrm{kbps}$ for a spreading factor of 32. In the downlink, the $\mathrm{CCH}$ and $\mathrm{DCH}$ can accommodate the maximum transport channel rate of $36 \mathrm{kbps}$ and $28.8 \mathrm{kbps}$ for a spreading factor of 64

\subsection{Radio Resources Allocations:}

Now better understanding from the UE (User Equipment) to camp into the network, the NBAP signalling exchanged between the L1 (Node B) and the collapsed RNC (Small cell architecture) are described essentially in two phases.

Initial phase of the UE attach procedure is captured below,

a. RADIO LINK SETUP REQUEST.

b. RADIO LINK SETUP RESPOSNE.

c. RADIO LINK RESTORE INDICATION.

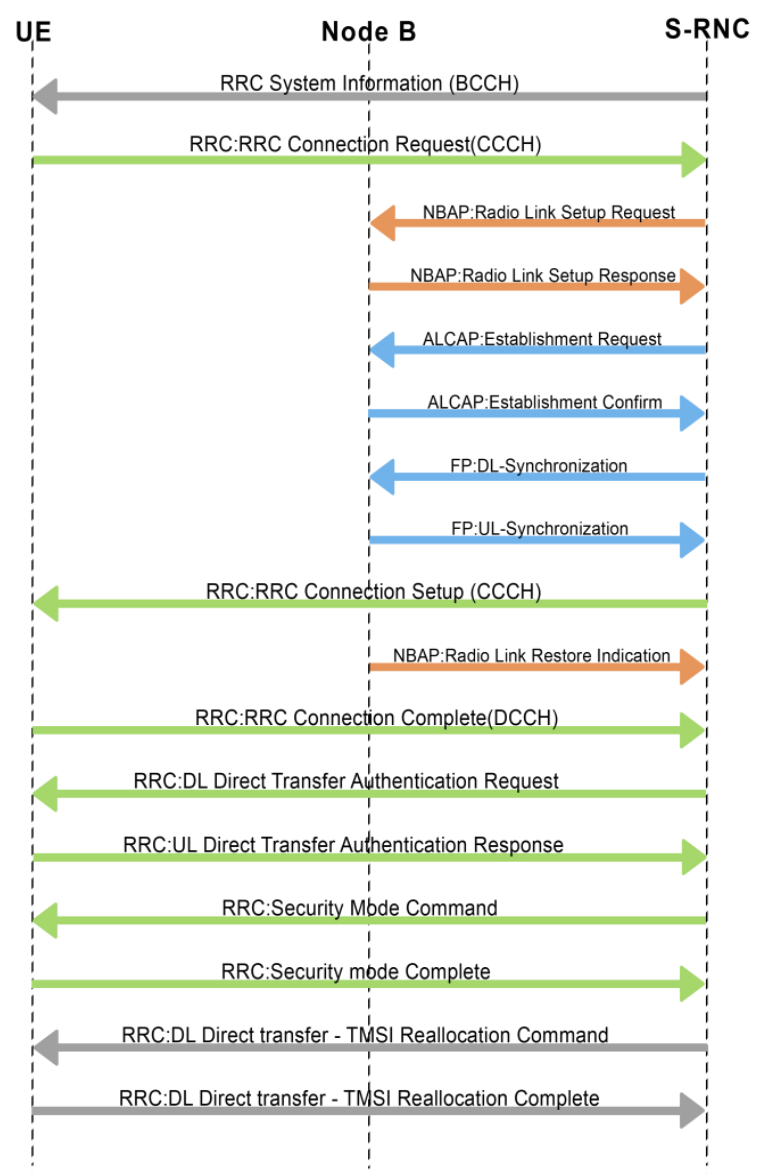

Figure 2: Phase1 procedural exchange of messages between User Equipment and the UMTS network elements.

The next phase involves the establishment of dedicated radio bearer for the Packet switched domain or Circuit switched domain pertains to NBAP (Node B Application Part) messages towards L1 (Node B) where the actually designated values for the establishment of required RAB and Quality of service which UE has asked for is intimated with a set of messages as prescribed below for better understanding.

\section{d. RADIO LINK RECONFIGURATION PREP REQUEST. e. RADIO LINK RECONFIGURATION READY. f. RADIO LINK RECONFIGURATION COMMIT.}

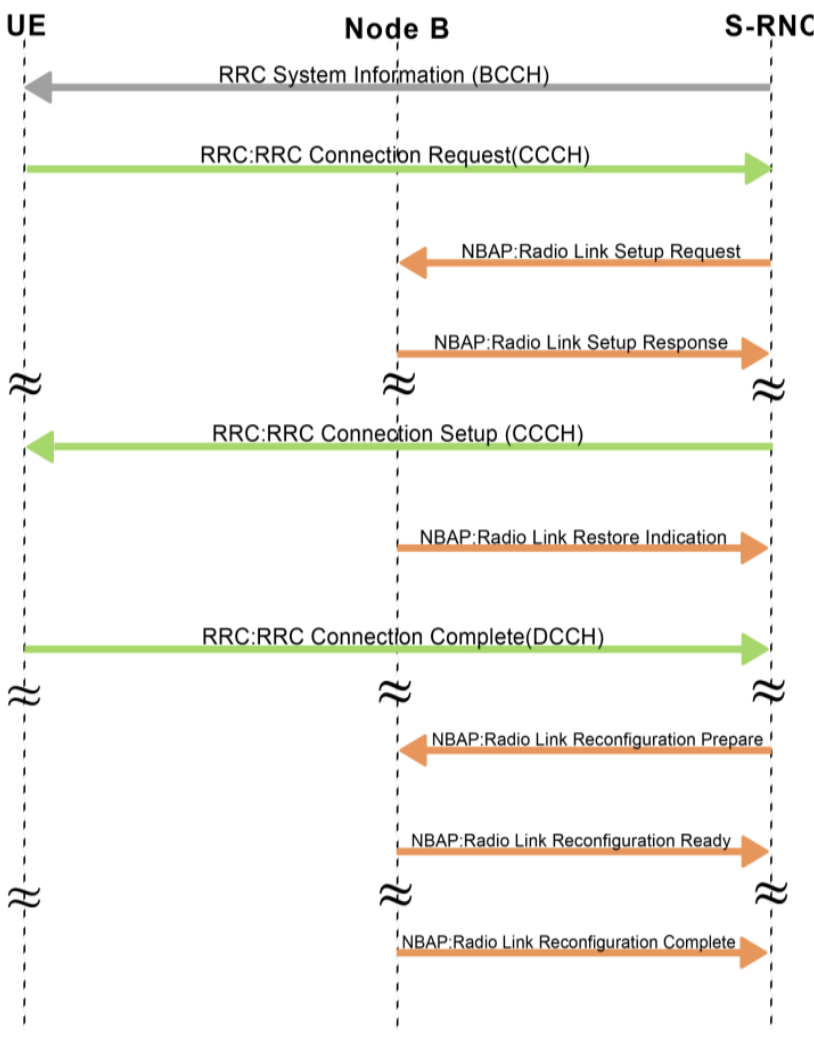

Figure 3: Phase2 procedural exchange of messages between User Equipment and the UMTS network elements.

The initial phase processing time is of 0.90929 seconds for the basic cell search, cellular camping towards the designed cell and corresponding NBAP (Node B Application Part) Signalling Radio Bearer establishment procedures per each UE and the subsequent phase (next phase) mentioned above consumes total time of 0.042508 seconds for the NBAP (Node B Application Part) Data Radio Bearer (Packet Switched Domain or Circuit Switched Domain) establishment procedures per each User Equipment (UE).

\section{PROBLEM STATEMENT}

\subsection{Reduced Signaling Procedures}

The latest generation smart phones in the serene tend to reduce the signaling message exchanges between UE and the cellular network. In the current scenario with the commercial UEs it's been noticed that when the UE is active with a Packet switched call (PS call) and is performing a periodic location update request procedure. If there is no response to the periodic location update (a CS domain activity) without giving an attempt of re-trying the Location update procedure again see the UE signals a signaling release of the PS domain thus reliving the active PS domain active connection to reselect the cell procedure. Since the PS domain release is executed, PDP Deactivation has to happen from the network system perspective, given that UE again attempts an PDP activate to resume the abruptly ended the Packet switched session which is an unexpected behaviour from UE side but an implementation specific performed outside of the scope of 3GPP standards. Due to the signalling release behaviour of 
UE a Radio Link Delete has to be triggered so that to honour the request form the UE and allow the UE to come back to the network cell to perform attach procedure to resume the upheld activities caused to loss of no response from the network for the periodic location update activity.

\subsection{Timing Reference}

The flow of exchange of NBAP messages between L1 and the RNC when the above described unexpected behavior occurs
i. RADIO LINK SETUP REQUEST.
ii. RADIO LINK SETUP RESPOSNE.
iii. RADIO LINK RESTORE INDICATION
iv. RADIO LINK RECONFIGURATION PREPARE REQUEST.
v. RADIO LINK RECONFIGURATION READY.
vi. RADIO LINK RECONFIGURATION COMMIT. $* * * * * * \mathrm{PS}$ call becomes active. $* * * * * * * * * * * * * *$ *******Periodic Location Update $* * * * * * * * * * * *$ ****** Signal Connection Release $* * * * * * * * * * *$
vii. RADIO LINK DELETE REQUEST. $* * * * * * \mathrm{RL}$ deleted due to reduced signaling Behavior of UE********************
viii. RADIO LINK SETUP REQUEST. ******New RL has to be established $* * * * * * * * * *$
ix. RADIO LINK SETUP RESPOSNE.
$x$. RADIO LINK RESTORE INDICATION.
xi. RADIO LINK RECONFIGURATION PREPARE REQUEST.
xii. RADIO LINK RECONFIGURATION READY. xiii. RADIO LINK RECONFIGURATION COMMIT.

As described above the time consumed (translates to MIBS from L1 perspective) for the completion of entire signaling exchanges from 1-6 takes close to $951.7 \mathrm{~ms}$ for a single UE and due to the unexpected behavior of UE the same procedure has to be repeated which would account the same time 951.7 ms giving lead to un-optimized execution of L1 and Higher layer application. The analysis of the above mentioned drop call probability factors with measured data obtained from an operative GSM network are presented. The data were collected over a period of three weeks for each of nine deployed commercial networks and comprised of the traffic intensity, drop-call rate, and number of call attempts. This translates to consumption of $1903.4 \mathrm{~ms}$ of processing time for a single UE. The interpretation of these results is that the network in question was well established and optimized and operating optimally during the period the measurements were taken.

\section{FALLBACK TO SIGNALING RADIO BEARER DESIGN APPROACH}

The novel method of handling this scenario is by effectively keeping the signaling radio bearer still active and just releasing the Packet switched connection as triggered by the UE. This gives room to shrink the signaling exchanges that happen between the UE and the Network there by saving upon lot of processing time (MIBS) as well as the battery life(UE perspective).
i. RADIO LINK SETUP REQUEST.
ii. RADIO LINK SETUP RESPOSNE.
iii. RADIO LINK RESTORE INDICATION.
iv. RADIO LINK RECONFIGURATION PREPARE REQUEST.
v. RADIO LINK RECONFIGURATION READY.
vi. RADIO LINK RECONFIGURATION COMMIT.

\author{
***********PS call becomes active. $* * * * * * * * * *$ \\ ***********Periodic Location Update $* * * * * * * *$ \\ $* * * * * * * * * *$ Signal Connection Release $* * * * * * *$ \\ vii. RADIO LINK RECONFIGURATION PREPARE \\ REQUEST. \\ $* * * * * *$ Configuration sent to release PS call \\ And retain SRB connection. $* * * * * * * * * *$ \\ viii. RADIO LINK RECONFIGURATION READY. \\ ix. RADIO LINK RECONFIGURATION COMMIT. \\ ******PS call gets released but the Radio Link \\ Is still active. $* * * * * * * * * * * * * * * *$ \\ $x$. RADIO LINK RECONFIGURATION PREPARE \\ REQUEST \\ **********New RL has to be established $* * * * * * *$ \\ xi. RADIO LINK RECONFIGURATION READY. \\ xii. RADIOLINK RECONFIGURATION COMMIT. \\ $* * * * * * * * * *$ PS call becomes active. $* * * * * * * * * *$
}

With this design we can effectively manage and support the commercial UE reduced signalling and efficient response activity with the below merits.

1) The redundant signalling exchange are waived off between the UE and the RNC thus enhancing the network performance of handling and retaining the UE back to desired Quality of service which was granted before the call drop/fail occurred.

2) The processing time to re-schedule the UE has been considerably reduced from $951.7 \mathrm{~ms}$ to $85.016 \mathrm{~ms}$ for each UE which results in highly optimized processing at both L1 (Node B) and RNC (Collapsed Architecture).

3) The battery life and the endurance of the UE to communicate with network for the service would be increased thus giving an advantage of better connectivity of the network services due the minimal signal exchange and efficient utilization of both the UE and Cellular Network resources.

\section{CONCLUSION}

In this paper, a technical insight has been provided with a simple analysis to study the impact of signaling load on call blocking/dropping. The calculation of the data rate requirement for basic signaling services are given in both common and dedicated radio channels with our suggested study of the coordination between voice and data transmissions in UMTS networks. Based on the study from a large carrier's data sets, we identify the features most relevant to the dropped calls from multiple measurements/features extracted from each call using machine learning techniques. $\mathrm{Fb}-\mathrm{SRB}$ provides an accurate and optimized solution as discussed in this paper. Fb-SRB does not need any additional investment as it can be mitigated at the software design overhaul at both Node B (L1) and Femtocell (RNC). The solution is applicable for 3GPP complaint Node B deployed in the telecom ecosystem which can be opted to improve the telecom infrastructure and minimize the call drop. Hence, improve the quality of service provided. It may help the telecom sector. Almost every telecom sector worldwide are upgrading their network infrastructure to reduce or minimize the problem of call drop with better KPI statics and network optimizations. The Fallback to SRB describes simple procedure for cellular network performance improvement. In this paper, it has been analytically proved that we can optimize and improvise an existing cellular network using updated methodology of Fall of SRB solution to fine parameter tuning to offer remarkable Quality of service to the 
end users. Moreover, the issue discussed here are quite helpful for the analysis and performance evaluation of different cellular networks. Optimization team use call drop reports in order to detect bad service quality areas. These reports also help to plan operators to enhance coverage, improve quality and increase capacity in the days to come. A mobile operator can also set its own targets based on the KPIs in order to ensure end user satisfaction. Quality of service reports based on different KPIs are duly beneficial for Management team to compare network performance with the competitor's one (called benchmarking) and to plan network evolution and strategy. Hence, during radio network planning and network upgrading, it is suggested to all mobile operators that they must divert attention towards better network dimensioning \& topology, allocated band scanning, traffic prediction \& modeling, network operational expense (OPEX), and network parameter settings to avoid subsequent issues during optimization phase. Secondly, end users require stringent service offered which compels cellular operators to optimize enhance network performance to meet revenue and commercial targets as well.

\section{REFERENCES}

[1] Shan Zhou, Jie Yang, etal. "Proactive Call Drop Avoidance in UMTS Networks",32nd IEEE Conference on Computer Communications, IEEE INFOCOM 2013 Turin, Italy, 2013, page(s): 425-429.

[2] Nathaniel S. Tarkaa, Joseph M. Mom, Cosmas I. Ani "Drop Call Probability Factors in Cellular Networks". International Journal of Scientific \& Engineering Research Volume 2, Issue 10, October-2011

[3] I. Tanaka, T. Koshimizu, and K. Nishida, "CS fallback function for combined LTE and $3 \mathrm{G}$ circuit switched services," NTT Docomo Technical Journal, vol. 11, no. 3, 2009, page(s): 13-19.

[4] Rajkiran. S. N and Arunlal. K. S, "Maximum Likelihood Trellis Decoder for Golay Codes with Puncturing and Shortening for MIMO in HSPA+", International Journal of Science and Research (IJSR), Volume 4 Issue 8, August 2015, on page(s):303-306.

[5] Y. Jin, N. Duffield, J. Erman, P. Haffner, S. Sen, and Z.L. Zhang, "A modular machine learning system for flowlevel traffic classification in large networks," ACM Trans. Knowl. Discov. Data, vol. 6, no. 1, Mar. 2012pp. $4: 1-4: 34$

[6] Saowaphak Sasanus and David Tipperl, "Impact of Signaling Load on the UMTS Call Blocking/Dropping", Telecommunications Program, School of Information Sciences University of Pittsburgh

[7] W. Song and W. Zhuang, "Multi-Service Load Sharing for Resource Management in the Cellular/WLAN Integrated Network," IEEE Transaction on Wireless Communication, 8, No 2., pp 725-735, Feb 2009.

[8] Prashant Shrivastava, Dr.(Mrs) Poonam Sinha," Reducing Call Drops Due to Cell Failure" International Journal Of Engineering And Computer Science ISSN:2319-7242 Volume - 5 Issue -02 February, 2016

[9] Udeh I.J, Offia I.S, Ibekwe Minimization of Call Drop In Code Division Multiple Access 2000IX in 3G Wireless Networks, International Journal of Scientific \& Engineering Research, Volume 5, Issue 10, October2014. 